\section{(6) OPEN ACCESS}

\title{
Treatment after traumatic shoulder dislocation: a systematic review with a network meta-analysis
}

\author{
Lauri Kavaja, 1,2 Tuomas Lähdeoja, 1,3,4 Antti Malmivaara, ${ }^{5,6}$ Mika Paavola ${ }^{4}$
}

- Additional material is published online only. To view please visit the journal online (http://dx.doi.org/10.1136/ bjsports-2017-098539).

${ }^{1}$ Medical Faculty, University of Helsinki, Helsinki, Finland ${ }^{2}$ Department of Surgery, South Carelia Central Hospital, Lappeenranta, Finland ${ }^{3}$ Finnish Center of Evidencebased Orthopaedics (FICEBO), University of Helsinki, Helsinki, Finland

${ }^{4}$ Department of Orthopaedics and Traumatology, Helsinki University Hospital, Töölö Hospital, Helsinki, Finland ${ }^{5}$ Centre for Health and Social Economics, Institute of Health and Welfare, Helsinki, Finland ${ }^{6}$ Orton Orthopaedic Hospital and Orton Research Institute, Orton Foundation, Helsinki, Finland

\section{Correspondence to}

Dr Lauri Kavaja, Medical Faculty, University of Helsinki, Helsinki 00290, Finland;

lauri.kavaja@helsinki.fi

LK and TL contributed equally.

Received 17 September 2017 Revised 8 May 2018 Accepted 16 May 2018 Published Online First 23 June 2018

\author{
ABSTRACT \\ Objective To review and compare treatments (1) \\ after primary traumatic shoulder dislocation aimed at \\ minimising the risk of chronic shoulder instability and (2) \\ for chronic post-traumatic shoulder instability. \\ Design Intervention systematic review with random \\ effects network meta-analysis and direct comparison \\ meta-analyses.
}

Data sources Electronic databases (Ovid MEDLINE, Cochrane Clinical Trials Register, Cochrane Database of Systematic Reviews, Embase, Scopus, CINAHL, Ovid MEDLINE Epub Ahead of Print, In-Process \& Other Non-Indexed Citations, Ovid MEDLINE Daily, DARE, HTA, NHSEED, Web of Science) and reference lists were searched from inception to 15 January 2018.

\section{Eligibility criteria for selecting}

studies Randomised trials comparing any interventions either after a first-time, traumatic shoulder dislocation or chronic post-traumatic shoulder instability, with a shoulder instability, function or quality of life outcome. Results Twenty-two randomised controlled trials were included. There was moderate quality evidence suggesting that labrum repair reduced the risk of future shoulder dislocation (relative risk $0.15 ; 95 \% \mathrm{Cl} 0.03$ to $0.8, p=0.026)$, and that with non-surgical management $47 \%$ of patients did not experience shoulder redislocation. Very low to low-quality evidence suggested no benefit of immobilisation in external rotation versus internal rotation. There was low-quality evidence that an open procedure was superior to arthroscopic surgery for preventing shoulder redislocations.

Conclusions There was moderate-quality evidence that half of the patients managed with physiotherapy after a first-time traumatic shoulder dislocation did not experience recurrent shoulder dislocations. If chronic instability develops, surgery could be considered. There was no evidence regarding the effectiveness of surgical management for post-traumatic chronic shoulder instability.

\section{INTRODUCTION}

The shoulder is the most commonly dislocated large joint. ${ }^{1}$ A traumatic shoulder dislocation is often accompanied by a labral lesion, ${ }^{2-7}$ which predisposes the patient to developing chronic shoulder instability. ${ }^{8-11}$ The incidence of primary shoulder dislocation varies between $15.3^{1}$ and $56.3^{12}$ per 100000 person-years. Most patients are men aged under 40 years and most dislocations are sports injuries. Both these facts make shoulder dislocation and possible subsequent chronic instability an important health issue for young, active patients and their treating clinicians. ${ }^{13}$
Acute treatment of a dislocated shoulder is closed reduction, which should be performed as soon as possible, either on the field or in an emergency department. ${ }^{14}$ Some patients develop recurrent dislocations or symptomatic subluxations even in daily activities. This has prompted suggestions that surgical stabilisation may be indicated after the first dislocation-a treatment strategy that has been investigated in several randomised controlled trials (RCT), with mixed results. ${ }^{34671516}$

There is considerable variation in the management of patients after a primary traumatic shoulder dislocation (both between surgeons and disciplines). ${ }^{17} 18$ Most patients with chronic post-traumatic shoulder instability are offered stabilisation surgery, while the surgical methods vary widely. ${ }^{17-20}$ Some evidence suggests that in the management of musculoskeletal conditions patients are more likely to undergo surgical treatment if the treating physician is a surgeon versus a non-surgical specialty (eg, physiotherapy), ${ }^{21}$ and that surgeons more easily recommend surgical treatment if the evidence regarding the effectiveness of surgery is inconclusive. ${ }^{19}$ Thus, to provide the best care, physiotherapists, physical medicine specialists, sports medicine specialists and orthopaedic surgeons should know what is the best quality evidence for treatment of shoulder instability.

Therefore, we aimed to assess the current evidence regarding the effectiveness of interventions after primary traumatic shoulder dislocation and in chronic post-traumatic shoulder instability. Our systematic review focused on two clinical effectiveness questions regarding post-traumatic shoulder instability: (1) What are the best treatments to reduce the incidence of chronic shoulder instability after a first-time traumatic shoulder dislocation? and (2) How can the clinician best treat a patient with chronic post-traumatic shoulder instability?

\section{METHODS}

We followed the Preferred Reporting Items for Systematic Reviews and Meta-Analyses statement ${ }^{22}$ when conducting and reporting this prospectively registered systematic review (PROSPERO registration ID: CRD42015020303). We use dislocation to refer to a true dislocation of the joint and instability to refer to dislocations, subluxations or other symptoms of instability either alone or together. By recurrent dislocation(s) and redislocation(s) we mean one or more dislocations after the primary episode, and by chronic post-traumatic instability we mean recurrent dislocation(s), subluxations or other symptoms of instability occurring after a firsttime traumatic shoulder dislocation. 


\section{Data sources and searches}

We searched Ovid MEDLINE, Cochrane Central Register of Controlled Trials, Cochrane Database of Systematic Reviews, Embase, Scopus, CINAHL, Ovid MEDLINE Epub Ahead of Print, In-Process \& Other Non-Indexed Citations, Ovid MEDLINE Daily, Database of Abstracts of Reviews of Effect (DARE), Health Technology Assessment Database, NHS Economic Evaluation Database and Web of Science on 15 January 2018. There were no restrictions on language or publication date. We adjusted the search strategies to meet the specifications of the individual databases (online supplementary appendix 1). We reviewed the reference lists of included publications and earlier reviews to identify any additional studies that were missed in the electronic database search. We also searched the WHO International Clinical Trials Registry Platform (WHO ICTRP) (www.who.int/ictrp) on 23 January 2018 for registrations of the included RCTs, possible completed but unpublished RCTs and ongoing RCTs.

\section{Study selection}

We included RCTs that compared any interventions after a first-time, traumatic shoulder dislocation or chronic post-traumatic shoulder instability with a follow-up of at least 1 year. We required an outcome measure related to shoulder instability, shoulder function or quality of life. We excluded studies of treatment of non-traumatic shoulder instability, cadaveric or biomechanical studies and studies on secondary shoulder instability (such as neurological conditions, syndromes or congenital conditions). Two investigators (LK and TL) independently assessed all identified publications for eligibility and resolved any disagreements by consensus.

\section{Data extraction and risk of bias assessment}

We categorised the included publications according to the two clinical scenarios under review (first-time traumatic shoulder dislocation and chronic post-traumatic shoulder instability). We extracted the outcome data for all available follow-up assessments. For primary shoulder dislocation, we used redislocation data from 1-year and 2-year follow-ups as defined in the individual studies. If numeric outcome data were unavailable, we extracted the data from figures and graphs.

In addition to the outcome measures, we extracted the following information regarding the trial characteristics and participants: study objectives, inclusion and exclusion criteria, number of patients allocated to intervention and control groups, follow-up time, sex distribution, mean age, proportion of sports injuries, indications for surgery, treatments for the intervention and control groups, associated soft-tissue and bony injuries, prespecified and reported harms, sample size estimations, study sponsorships and conflict of interest statements and trial registry identifiers. We extracted the method of randomisation and randomisation sequence generation, allocation concealment, degrees of blinding, loss to follow-up, intention-to-treat analysis, selective reporting, similarity of patient groups, cointerventions, compliance and timing of the outcome assessment. All data were extracted to a customised worksheet independently by two reviewers (LK and TL). We sent emails to authors of potentially eligible conference abstracts, publications with no available source data and unpublished trials and inquired about the status of the trial and requested data to be included in the meta-analyses.

Two reviewers (LK and TL) independently assessed the studylevel risk of bias according to the guidelines of the Cochrane Back Review Group. ${ }^{23}$ The risk of bias assessment has 12 independent criteria; with a judgement of 'yes', 'unclear' or 'no'. If at least six of the 12 criteria were judged to be at low risk of bias ('yes'), the risk of bias in the publication was considered low. ${ }^{23}$

To assess possible selective reporting of results, we compared the outcome measures specified in the methods section of the publication and in the trial registry (if available) to what was reported in the results section of the publication. For the analysis of the timing of the outcome assessment, we deemed a deviation of 3 months (in a 2-year follow-up) to be an acceptable range. Disagreements between the two reviewers on the retrieved data were resolved by consensus. If any item was unclear, we contacted the authors by email to clarify the issue.

Researcher conflict of interest and industry sponsorship have been identified as potential sources of bias. ${ }^{24-26}$ We evaluated and noted the existence of these potential sources of bias in the studies.

\section{Data synthesis and analysis}

We performed meta-analyses of clinically homogenous studies (patient populations, indications of treatment, intervention pairs and outcomes). If studies with same intervention-control pairs were not sufficiently homogenous, we performed multiple pairwise meta-analyses of the trials with poolable patients and data. In the analysis of surgical treatment after first-time shoulder dislocation, we performed a frequentist network meta-analysis to assess the effectiveness of surgical treatment alternatives at matching time points available in the source data. We prioritised outcomes at 2 years since the majority of shoulder redislocations take place within that time. ${ }^{27-33}$ If a trial had reported outcomes at multiple time points, we used data from one time point only in a single analysis. We calculated the number needed to treat (NNT) and number needed to harm $(\mathrm{NNH})^{34}$ for interventions with dichotomous outcomes and statistically significant betweengroup comparisons. For shoulder redislocations, the NNT is the number of patients who need to receive a treatment to prevent one patient having a shoulder redislocation. The $\mathrm{NNH}$ is the number of patients who need to be treated in a certain way to cause a redislocation. For NNT estimates we used the point estimate of the relevant risk ratio, base redislocation risk from a systematic review (21\%-47\%, minimum follow-up of 2 years $)^{35}$ and the redislocation rates in the relevant included trials.

We based all analyses on random effects models due to expected variation among patients and outcome measurements. Statistical heterogeneity was quantified with the $\mathrm{I}^{2}$ statistic. Sensitivity analyses or metaregression was not performed to explore heterogeneity or the effect of bias. In the network meta-analysis (NMA), the assumption of exchangeability was evaluated qualitatively. Inconsistency was evaluated using tau ${ }^{2}$ and Cochran $Q$ statistic and corresponding $\mathrm{p}$ values, and the Netheat plot. ${ }^{36}$ Use of quantitative methods to assess exchangeability or the use of p score to estimate the best treatment was not possible due to the low number of trials in the network. We did not plan subgroup analyses.

We used the Grading of Recommendations Assessment, Development and Evaluation (GRADE) approach to rate the quality of evidence and present a summary of findings table. ${ }^{37}$ We downgraded for inconsistency if the magnitude or direction of effects were dissimilar, the CIs had minimal overlap, the test of heterogeneity was significant or if the $\mathrm{I}^{2}$ was $>50 \%$. For imprecision, we downgraded if the CIs were very wide or if the Optimal Information Size criteria were not met. We used Review Manager (RevMan) (V.5.3.5) ${ }^{38}$ for the meta-analyses, the netmeta package $^{36}$ in $\mathrm{R}(\mathrm{V} .3 .2 .1)^{39}$ for the network meta-analysis and 

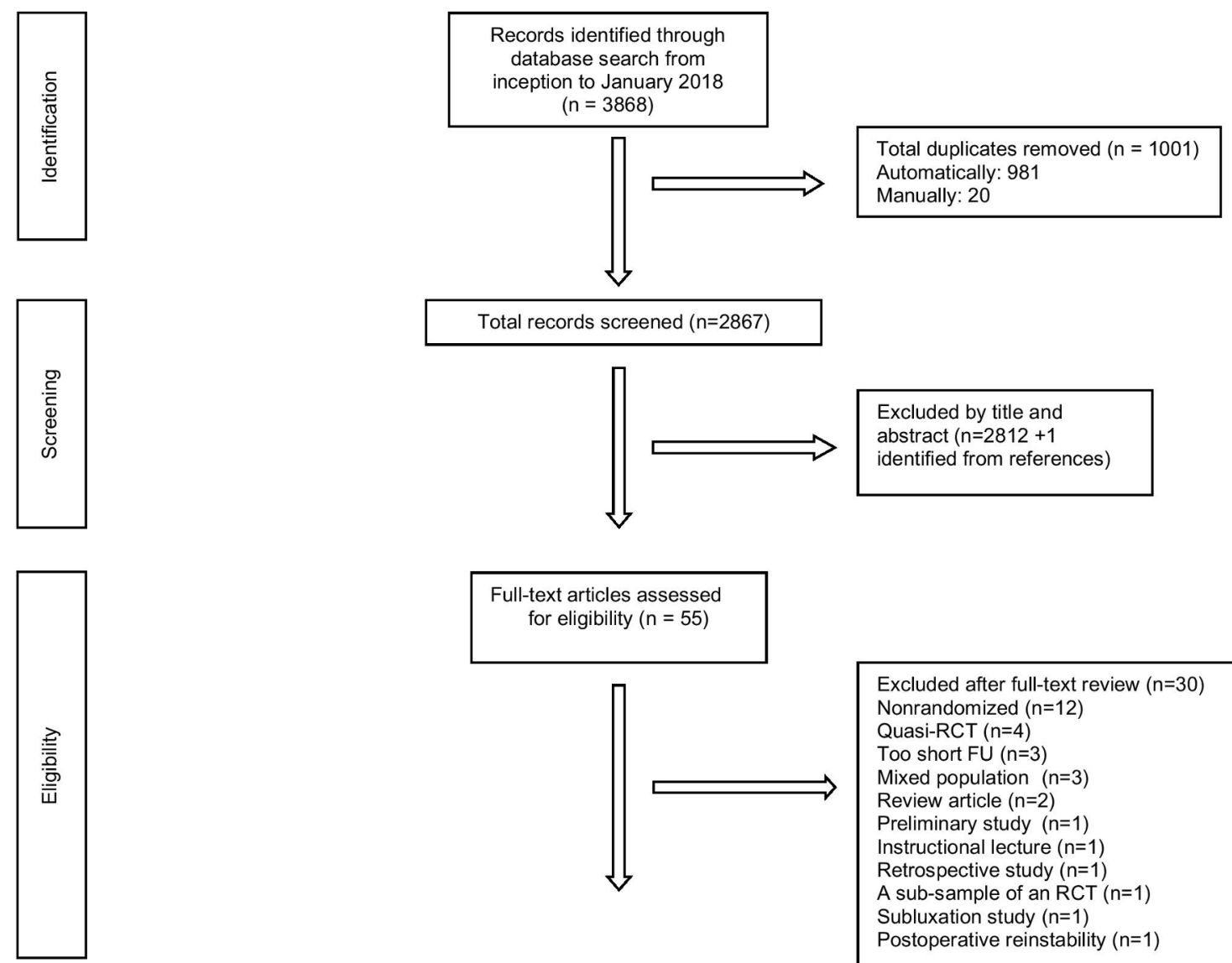

Full-text articles assessed for eligibility $(n=55)$

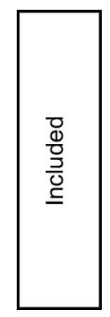

Included articles $\left(\mathrm{n}=25^{\star}\right)$

Excluded after full-text review $(n=30)$

Nonrandomized $(n=12)$

Quasi-RCT $(n=4)$

Too short FU $(n=3)$

Mixed population $(n=3)$

Review article $(n=2)$

Preliminary study $(n=1)$

Instructional lecture $(n=1)$

Retrospective study $(n=1)$

A sub-sample of an RCT $(n=1)$

Subluxation study $(n=1)$

Postoperative reinstability $(n=1)$
GRADEpro GDT ${ }^{40}$ for the GRADE judgements and summary of findings tables.

\section{Patient involvement}

The study did not involve patients.

\section{RESULTS}

The literature search yielded 2867 reports after exclusion of duplicates. Of these reports, 55 were considered for inclusion after review of titles and abstracts (figure 1). After full-text review, 25 publications (22 RCTs) were included (figure 1). Studies excluded at the full-text screening stage are presented in online supplementary appendix table 1 . We identified six unpublished RCTs (online supplementary appendix table 2). Five of these explored external rotation (ER) versus internal rotation (IR) as a treatment for a first-time traumatic shoulder dislocation. We did not receive data of any trial. We identified 20 ongoing RCTs (online supplementary appendix table 3 ).

Of the 22 included RCTs, 10 investigated treatment after a first-time traumatic shoulder dislocation. Four studied whether early surgery was beneficial after a first-time traumatic shoulder dislocation, 34671516 and five compared the effect of upper limb immobilisation in ER versus IR in preventing further instability. ${ }^{41-45}$ Twelve RCTs focused on chronic post-traumatic shoulder instability, of which four RCTs compared open labral repair with arthroscopic labral repair ${ }^{25467}$ and three compared absorbable with non-absorbable suture anchors. ${ }^{48-50}$

Mean participant age ranged from 20.3 to 36 years. The mean follow-up ranged from 12 to 143 months. In 15 RCTs, 67\% of the primary dislocations were sports injuries; seven RCTs did not report injury mechanism. ${ }^{2} 5454650-52$ One study included patients with large bony defects of the glenoid. ${ }^{52}$ The trials are summarised in table 1 ; online supplementary appendix tables 4 and 5 present all extracted data.

\section{Risk of bias assessment}

All trials were of parallel-group, prospective design. Twenty-one were classical RCTs; one used a minimisation algorithm. ${ }^{6}$ Two trials were double blind, ${ }^{649}$ seven were single blind ${ }^{4} 1545485153-56$ and 13 were not blinded. ${ }^{23571641-444647505257}$ Loss to follow-up ranged from $0 \%$ to $20 \%$. Intention-to-treat analysis was reported in all publications. The sample sizes ranged from 30 to 198 participants. Sample size estimation was described by 14 trials ${ }^{4-61541-45474852-545657}$; one of these met the prespecified 
Table 1 Summary of included studies

\begin{tabular}{|c|c|c|c|c|c|c|}
\hline Author, year, country & Intervention & Control & $\begin{array}{l}\mathrm{N}: \text { recruited/ } \\
\text { FU }\end{array}$ & Follow-up time & $\begin{array}{l}\text { Mean age of } \\
\text { patients }\end{array}$ & Main outcome (primary outcome if defined) \\
\hline \multicolumn{7}{|l|}{ Early surgery } \\
\hline $\begin{array}{l}\text { Jakobsen et al }(2007)^{3} \\
\text { Denmark }\end{array}$ & $\begin{array}{l}\text { DA and open } \\
\text { LR }\end{array}$ & $\begin{array}{l}\text { Arthroscopic } \\
\text { lavage }\end{array}$ & $76 / 75$ & $\begin{array}{l}2 \text { years } \\
10 \text { years }\end{array}$ & $\begin{array}{l}\text { I: } 23(15-39) \\
\text { C: } 20(15-31)\end{array}$ & $\begin{array}{l}\text { RDR } 2 \text { years: I: } 1 / 37(3 \%)-C: 21 / 39 \\
(54 \%), p=0.0011 \\
\text { RDR } 10 \text { years I: } 3 / 36(9 \%)-C: 24 / 39(62 \%), p \text { not } \\
\text { reported }\end{array}$ \\
\hline Finestone et al (2009) ${ }^{41}$ Israel & ERI 4 weeks & IRI 4 weeks & $51 / 51$ & $\begin{array}{l}\text { I: } 35.8(24-48) \\
\text { C: } 30.8(24-47)\end{array}$ & 20.3 & RDR: I: $10 / 27(37 \%)-C: 10 / 24(42 \%)$, NS \\
\hline $\begin{array}{l}\text { Liavaag et al }(2011)^{44} \\
\text { Norway }\end{array}$ & ERI 3 weeks & IRI 3 weeks & $188 / 183$ & $29.1(24-54)$ & $\begin{array}{l}26.8(15.9- \\
40, \pm 7.1)\end{array}$ & $\begin{array}{l}\text { RDR: I: } 28 / 91(31 \%)-C: 23 / 93(25 \%) \text {, NS } \\
\text { RIR: I: } 31 / 81(38 \%) — C: 36 / 82(42 \%), \text { NS }\end{array}$ \\
\hline Heidari et al (2014) ${ }^{42}$ Iran & ERI 3 weeks & IRI 3 weeks & $102 / 102$ & 24 & $\begin{array}{l}\text { I: } 36( \pm 7.8) \\
\text { C: } 35.43( \pm 10.0)\end{array}$ & RIR: I: $2 / 51(3.9 \%) — C: 17 / 51(33.3 \%), p<0.001$ \\
\hline $\begin{array}{l}\text { Whelan et al }(2014)^{45} \\
\text { Canada }\end{array}$ & ERI 4 weeks & IRI 4 weeks & $60 / 50$ & $25(12-43)$ & $\begin{array}{l}\text { I: } 23(16-35) \\
\text { C: } 23(14-34)\end{array}$ & RDR: I: $6 / 27(22 \%) — C: ~ 8 / 25(32 \%)$, NS \\
\hline \multicolumn{7}{|l|}{ Use of restriction band } \\
\hline Itoi et al $(2013)^{57}$ Japan & $\begin{array}{l}\text { C+11: MRB } 3 \\
\text { or I2: MRB } 6 \\
\text { weeks }\end{array}$ & ERI 3 weeks & $109 / 90$ & $\begin{array}{l}11: 26.5,12: 26.5 \\
C: 25.5\end{array}$ & $30(15-84)$ & $\begin{array}{l}\text { RDR: I1: } 10 / 31(32 \%) — 12: 10 / 30(33 \%) — C: 8 / 29 \\
(28 \%) \text {, NS }\end{array}$ \\
\hline $\begin{array}{l}\text { Archetti Netto et al }(2012)^{5} \\
\text { Brazil }\end{array}$ & $\begin{array}{l}\text { Arthroscopic } \\
\text { LR }\end{array}$ & Open LR & $50 / 42$ & $37.5(20-56)$ & $\begin{array}{l}\text { I: } 27.5( \pm 5.4) \\
\text { C: } 30.8( \pm 5.6)\end{array}$ & $\begin{array}{l}\text { DASH (range, SD): I: } 2.65(0-24, \pm 7.3)-C: 4.22 \\
(0-21, \pm 5.8), p=0.031 \text { (MCID is }>10)^{91}\end{array}$ \\
\hline $\begin{array}{l}\text { Mohtadi et al (2014) } \\
\text { Canada }\end{array}$ & Open LR & Arthroscopic LR & $196 / 162$ & 24 & $\begin{array}{l}\text { I: } 27.8(16- \\
53.7, \pm 7.9) \\
\text { C: } 27.2(16.5- \\
59, \pm 9)\end{array}$ & $\begin{array}{l}\text { WOSI }(95 \% \mathrm{CI}) \mathrm{BL} \rightarrow \mathrm{FU}: \mathrm{I}: 41.7 \%(37.9-45.5) \rightarrow \\
85.2 \%(80.5-89.8)-\mathrm{C}: 40.6 \%(36.9-44.3) \rightarrow \\
81.9 \%(77.4-86.4), \text { NS }\end{array}$ \\
\hline \multicolumn{7}{|c|}{ Absorbable or non-absorbable implant materials (anchors) } \\
\hline Warme et al (1999) ${ }^{50}$ USA & $\begin{array}{l}\text { Open LR, } \\
\text { non-A SA }\end{array}$ & $\begin{array}{l}\text { Open LR, } \\
\text { bio-A SA }\end{array}$ & $38 / 40$ & $25(17-45)$ & $22(17-46) \dagger$ & Loss of ER $\left(^{\circ}\right)$ : I: $3(0-15)-C: 3(0-10)$, NS \\
\hline Tan et al $(2006)^{49}$ UK & $\begin{array}{l}\text { Arthroscopic } \\
\text { LR, non-A SA }\end{array}$ & $\begin{array}{l}\text { Arthroscopic LR, } \\
\text { bio-A SA }\end{array}$ & $124 / 124$ & $2.6(1.5-5)$ years & $\begin{array}{l}\text { I: } 27(18-45, \pm 7) \\
\text { C: } 28(17-49, \pm 8)\end{array}$ & $\begin{array}{l}\text { OSIS (SD): BL } \rightarrow \text { FU: I: } 36( \pm 8) \rightarrow 18( \pm 6)-C: 36 \\
( \pm 7) \rightarrow 20( \pm 10), \text { p not reported }\end{array}$ \\
\hline Milano et al $(2010)^{48}$ Italy & $\begin{array}{l}\text { Arthroscopic } \\
\text { LR, non-A SA }\end{array}$ & $\begin{array}{l}\text { Arthroscopic LR, } \\
\text { bio-A SA }\end{array}$ & $78 / 70$ & $24.5(22-29)$ & $\begin{array}{l}\text { I: } 28(16-46) \\
\text { C: } 28(16-52)\end{array}$ & DASH (range): I: 4.5 (0-27)—C: 7 (0-25), NS \\
\hline \multicolumn{7}{|c|}{ Addition of posterior capsular plication } \\
\hline Castagna et al $(2009)^{51}$ Italy & $\begin{array}{l}\text { Arthroscopic } \\
\text { LR, bio-A SA }\end{array}$ & $\begin{array}{l}\text { I+posterior } \\
\text { 2-anchor } \\
\text { capsular } \\
\text { plication }\end{array}$ & $40 / 40$ & 2 years & $\begin{array}{l}\text { I: } 29.1 \\
\text { C: } 27.3\end{array}$ & $\begin{array}{l}\mathrm{FF} \mathrm{BL} \rightarrow \text { FU: I: } 169(83-105) \rightarrow 172.5(155-180)- \\
\mathrm{C:} 177.8(170-180) \rightarrow 163.3(140-175) \mathrm{p} \text { for } \\
\text { change }<0.001\end{array}$ \\
\hline \multicolumn{7}{|c|}{ Different absorbable implant materials } \\
\hline $\begin{array}{l}\text { (I) Magnusson et al }(2006)^{53} \\
\text { Sweden } \\
\text { (II) Elmlund et al }(2009)^{54} \\
\text { Sweden }\end{array}$ & $\begin{array}{l}\text { Arthroscopic } \\
\text { LR PLLA tacks }\end{array}$ & $\begin{array}{l}\text { Arthroscopic LR } \\
\text { PGACP tacks }\end{array}$ & $40 / 35$ & $\begin{array}{l}\text { (I) I: } 25(24-34) \\
\text { C: } 26(23-35) \\
\text { (II) I: } 80(75-95) \\
\text { C: } 81 \text { (64-96) }\end{array}$ & $\begin{array}{l}\text { I: } 26(16-50) \ddagger \\
\text { C: } 30(15-45) \ddagger\end{array}$ & $\begin{array}{l}\text { (I) Drill hole visibility: more visible in I, } p<0.004 \\
\text { (II) Drill hole visibility: more visible in I, } p<0.0001\end{array}$ \\
\hline ssorbable or non-absorbable & suture material & & & & & \\
\hline
\end{tabular}




\begin{tabular}{|c|c|c|c|c|c|c|}
\hline Author, year, country & Intervention & Control & $\begin{array}{l}\text { N: recruited/ } \\
\text { FU }\end{array}$ & Follow-up time & $\begin{array}{l}\text { Mean age of } \\
\text { patients }\end{array}$ & Main outcome (primary outcome if defined) \\
\hline Monteiro et al $(2008)^{55}$ Brazil & $\begin{array}{l}\text { Arthroscopic } \\
\text { LR bio-A } \\
\text { SA+bio-A } \\
\text { sutures }\end{array}$ & $\begin{array}{l}\text { Arthroscopic } \\
\text { LR bio-A } \\
\text { SA+non-A } \\
\text { sutures }\end{array}$ & $50 / 45$ & $\begin{array}{l}\text { I: } 31.5(24-45) \\
\text { C: } 30.9(24-45)\end{array}$ & $23.5(16-37)$ & 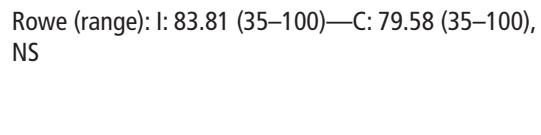 \\
\hline \multicolumn{7}{|l|}{ Rehabilitation } \\
\hline Kim et al $(2003)^{56}$ Korea & Accelerated & Traditional & $62 / 62$ & $31(27-45, \pm 9)$ & $\begin{array}{l}\text { l: } 29(15- \\
38, \pm 5.8) \neq \\
\text { C: } 28(18- \\
39, \pm 5.6) \ddagger\end{array}$ & RDR: 0/62 (0\%), subluxation rate: 0/62 (0\%), NS \\
\hline \multicolumn{7}{|c|}{ Anatomic versus non-anatomic surgical techniques } \\
\hline $\begin{array}{l}\text { Salomonsson et al } \\
(2009)^{52} \text { Sweden }\end{array}$ & $\begin{array}{l}\text { Open LR } \\
\text { and capsular } \\
\text { imbrication }\end{array}$ & $\begin{array}{l}\text { Modified Putti- } \\
\text { Platt procedure }\end{array}$ & $66 / 62$ & $143(121-162, \pm 12.2)$ & $\begin{array}{l}\text { I: } 29(17-52) \ddagger \\
\text { C: } 26(16-63) \ddagger\end{array}$ & Rowe: I: 90, C: 90, NS \\
\hline
\end{tabular}

Follow-up times are tabulated as presented in the original publication as mean (range) in months unless noted otherwise. Mean age is presented as mean (range) or mean ( \pm SD) or mean (range $\pm \mathrm{SD}$ ) in years unless noted otherwise. The point of recording participant age was not specified in the publication, unless marked otherwise (*at first dislocation, tat surgery, łpreoperatively).

Bio-A, bio-absorbable; BL, base level; C, control; CS, constantscore; DA, diagnostic arthroscopy; DASH, disabilities of the arm, shoulder and hand; ER, external rotation; ERI, external rotation immobilisation; FF, forward flexion (in degrees); FU, follow-up; I, intervention; IRI, internal rotation immobilisation; LR, labrum repair; MCID, minimal clinically important difference; MRB, motion restriction band; non-A, non-absorbable; NS, no statistically significant difference; OISS, Oxford Instability Shoulder Score; PGACP, polygluconate co-polymer; PLLA, polylactic acid; RDR, redislocation rate; RIR, recurrent instability rate; SA, suture anchor; WOSI, Western Ontario Shoulder Instability Index Score.

assumptions of the sample size estimation. ${ }^{42}$ Levels of evidence of the included trials are presented in online supplementary appendix table 6 .

Risk of bias was high in two RCTs. ${ }^{41} 50$ There was a high risk of detection bias due to variation in the timing of the follow-up assessments; three ${ }^{43448}(14 \%)$ of the 22 trials met the minimum criterion of less than 3-month discrepancy between scheduled and actual follow-up visit (online supplementary appendix table 7).

Two trials disclosed a potential conflict of interest and industry sponsorship, ${ }^{54} 57$ three disclosed industry sponsorship 435055 and five disclosed either conflict of interest or industry sponsorship. ${ }^{3-5} 5253$ Six trials made an explicit statement of no conflicts of interest or industry sponsorship, ${ }^{6414244447}$ and nine did not mention conflict of interest or industry sponsorship (online supplementary appendix table 7). ${ }^{2715164648495156}$

\section{GRADE analysis and summary of findings}

The quality of evidence varied from very low to moderate. The most common reasons for downgrading were absence of blinding and imprecision. Summary of findings tables are presented below within the subsections of synthesis of results.

\section{Synthesis of results}

First-time traumatic shoulder dislocation

Early surgery

Patients who underwent surgery to repair a labral injury (ie, Bankart repair) had fewer redislocations than patients who received non-surgical management or arthroscopic lavage of the glenohumeral joint at 1year (four studies, 273 patients) ${ }^{367}$ (relative risk (RR) labrum repair vs non-surgical management 0.08 ; 95\% CI 0.02 to $0.27, \mathrm{p}<0.001$ and labrum repair vs arthroscopic lavage $0.23 ; 95 \%$ CI 0.08 to $0.67, \mathrm{p}=0.007, \mathrm{I}^{2}=0 \%, \mathrm{p}=0.46$ ) and 2 years (four studies, 243 patients) ${ }^{34616}$ (RR labrum repair vs non-surgical
A

$\begin{array}{ll}\text { Treatment } \mathbf{1} & \\ \text { Labrum repair } & \text { vs } \\ \text { Labrum repair } & \text { vs } \\ \text { Lavage } & \text { vs }\end{array}$

Lavage vs

$\begin{array}{rr}\text { Treatment 2 } & \text { Relative risk }(95-\% \mathrm{Cl}) \\ \text { NOM } & 0.08(0.02,0.27) \\ \text { Lavage } & 0.23(0.08,0.67) \\ \text { NOM } & 0.34(0.14,0.86)\end{array}$

$0.34(0.14,0.86)$

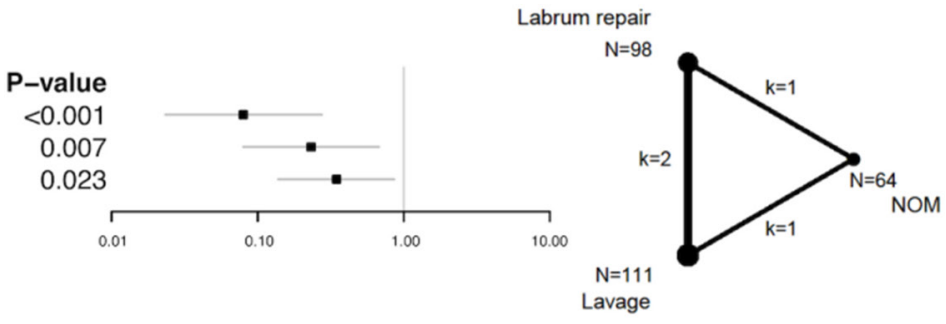

B

Treatment 1

Labrum repair vs

Labrum repair vs

Lavage

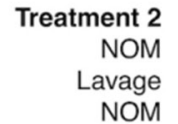

$<0.001$

0.023

Figure 2 Relative risk (RR) of a redislocation after treatment of a first-time traumatic shoulder dislocation at (A) 1 year and at (B) 2 years derived from the network meta-analysis. 
Table 2 Summary of findings for labrum repair after a first-time traumatic shoulder dislocation

\begin{tabular}{|c|c|c|c|c|c|c|}
\hline \multicolumn{7}{|c|}{ Labrum repair compared with physiotherapy for prevention of recurrent dislocations after a first-time traumatic shoulder dislocation } \\
\hline \multicolumn{7}{|c|}{$\begin{array}{l}\text { Patient or population: a first-time traumatic shoulder dislocation in general population } \\
\text { Setting: surgical versus non-surgical treatment } \\
\text { Intervention: labrum repair } \\
\text { Comparison: physiotherapy }\end{array}$} \\
\hline \multirow{2}{*}{$\begin{array}{l}\text { Outcome } \\
\text { Number of participants } \\
\text { (studies) }\end{array}$} & \multirow{2}{*}{$\begin{array}{l}\text { Relative effect } \\
(95 \% \mathrm{Cl})\end{array}$} & \multicolumn{3}{|c|}{ Anticipated absolute effects $(95 \% \mathrm{Cl})$} & \multirow[b]{2}{*}{ Certainty } & \multirow[b]{2}{*}{ What happens } \\
\hline & & Non-surgical & Labrum repair & Difference & & \\
\hline $\begin{array}{l}\text { Redislocation rate after surgical treatment } \\
\text { of primary shoulder dislocation } \\
\text { Follow-up: mean } 2 \text { years } \\
\text { Number of participants: } 243 \\
\text { (four RCTs) }\end{array}$ & $\begin{array}{l}\text { RR } 0.15 \\
(0.03 \text { to } 0.8)\end{array}$ & $52.9 \%$ & $\begin{array}{l}7.9 \% \\
(1.5 \text { to } 42.3)\end{array}$ & $\begin{array}{l}45 \% \text { fewer } \\
\text { (51.4 fewer to } 10.6 \\
\text { fewer) }\end{array}$ & $\begin{array}{l}\oplus \oplus \oplus \bigcirc \\
\text { Moderate* }^{*}\end{array}$ & $\begin{array}{l}\text { Labrum repair may be } \\
\text { beneficial in reducing the } \\
\text { rate of redislocations. }\end{array}$ \\
\hline
\end{tabular}

${ }^{*}$ Rated down for serious risk of bias (lack of blinding) and serious imprecision. Rated up for large magnitude of an effect.

$\mathrm{RCT}$, randomised controlled trial; $\mathrm{RR}$, relative risk.

Explanations for the Cochrane Summary of Findings table (http://www.cochranelibrary.com/about/explanations-for-cochrane-summary-of-findings-sof-tables.html)

management $0.15 ; 95 \% \mathrm{CI} 0.03$ to $0.8, \mathrm{p}=0.026$ and labrum repair vs arthroscopic lavage RR $0.21 ; 95 \% \mathrm{CI} 0.05$ to $0.91, \mathrm{p}=0.037$, $\mathrm{I}^{2}=63.6 \%, \mathrm{p}=0.064$ ) (figure $2 \mathrm{~A}, \mathrm{~B}$; table 2). There was a benefit of arthroscopic lavage at 1 year over non-surgical management (RR 0.34; 95\% CI 0.14 to $0.86, \mathrm{p}=0.023$ ) (figure $2 \mathrm{~A}$ ); this effect was not evident at 2 years of follow-up (RR $0.71 ; 95 \%$ CI 0.14 to $3.68, \mathrm{p}=0.686$ ) (figure 2B). Tests for inconsistency were insignificant at both time points. Direct meta-analysis was possible between studies comparing labrum repair and arthroscopic lavage (two studies, 160 patients). ${ }^{36}$ There was a treatment effect in favour of labrum repair (RR $0.13 ; 95 \% \mathrm{CI} 0.03$ to $0.69, \mathrm{p}=0.02, \mathrm{I}^{2}=54 \%$, $\mathrm{p}=0.14$ ) (online supplementary appendix figure 1 ). The NNT to prevent a redislocation at 2 years ranged from 2.5 to 5.6 based on external data ${ }^{35}$ and 2.0 and $4.7^{346}$ using the redislocation rates in the included RCTs.

\section{Arm position}

Immobilisation in ER (vs IR) had no effect on the redislocations or chronic instability (table 3 ). Due to heterogeneity of the trials, three separate direct comparison meta-analyses comparing immobilisation in ER versus IR were performed: one of trials with young patients and narrow age range reporting redislocations, second of a subset of the former reporting instability and a third of older patients with a wide age range reporting instability. In none did the arm position affect the outcome. Three trials (287 patients) ${ }^{4144}$ had a narrow age range with an average age of approximately 25 years reporting redislocations (RR $1.07 ; 95 \%$ CI 0.76 to $1.50 ; \mathrm{p}=0.70 ; \mathrm{I}^{2}=0 \% ; \mathrm{p}=0.65$ ) (online supplementary appendix figure $2 \mathrm{a}$ ); two of these trials (236 patients) ${ }^{44} 45$ also reported recurrent instability (RR 1.01; $95 \%$ CI 0.82 to $1.24 ; \mathrm{p}=0.90 ; \mathrm{I}^{2}=0 \% ; \mathrm{p}=0.78$ ) (online supplementary appendix figure $2 \mathrm{~b}$ ). Two trials (261 patients $)^{42} 43$ had a wide age range with an average age of approximately 35 years and reported recurrent instability (RR $0.31 ; 95 \%$ CI 0.06 to $1.68 ; \mathrm{p}=0.17 ; \mathrm{I}^{2}=82 \% ; \mathrm{p}=0.02$ ) (online supplementary appendix figure $2 c$ ).

Table 3 Summary of findings for immobilisation in external versus internal rotation after a first-time traumatic shoulder dislocation

Immobilisation in external rotation compared with internal rotation for prevention of recurrent dislocations or chronic instability after a first-time traumatic shoulder dislocation

\begin{tabular}{|c|c|c|c|c|c|c|}
\hline $\begin{array}{l}\text { Patient or population: a first-time traumatic s } \\
\text { Setting: non-surgical versus non-surgical man } \\
\text { Intervention: immobilisation in external rotat } \\
\text { Comparison: immobilisation in internal rotati }\end{array}$ & $\begin{array}{l}\text { houlder dislocatio } \\
\text { agement } \\
\text { ion } \\
\text { on }\end{array}$ & $n$ in gen & opulation & & & \\
\hline $\begin{array}{l}\text { Number of participants } \\
\text { (studies) }\end{array}$ & $\begin{array}{l}\text { Relative effect } \\
(95 \% \mathrm{Cl})\end{array}$ & IR & ER & Difference & Certainty & What happens \\
\hline $\begin{array}{l}\text { Recurrent instability rate after immobilisation in } \\
\text { treatment of primary shoulder dislocation in older } \\
\text { population } \\
\text { Follow-up: mean } 2 \text { years } \\
\text { Number of participants: } 261 \\
\text { (two RCTs) }\end{array}$ & $\begin{array}{l}\text { RR } 0.31 \\
\text { (0.06 to } 1.68)\end{array}$ & $38.4 \%$ & $\begin{array}{l}11.9 \% \\
(2.3 \text { to } 64.5)\end{array}$ & $\begin{array}{l}\mathbf{2 6 . 5 \%} \text { fewer } \\
\text { (36.1 fewer to } 26.1 \text { more) }\end{array}$ & $\begin{array}{l}\oplus \bigcirc \bigcirc \bigcirc \\
\text { Very low* }\end{array}$ & $\begin{array}{l}\text { Immobilisation in external } \\
\text { rotation does not seem to be } \\
\text { beneficial in prevention of } \\
\text { shoulder instability. }\end{array}$ \\
\hline $\begin{array}{l}\text { Redislocation rate after immobilisation in } \\
\text { treatment of primary shoulder dislocation in } \\
\text { younger population } \\
\text { Follow-up: mean } 2 \text { years } \\
\text { Number of participants: } 287 \\
\text { (three RCTs) }\end{array}$ & $\begin{array}{l}\text { RR } 1.07 \\
\text { (0.76 to } 1.50)\end{array}$ & $30.3 \%$ & $\begin{array}{l}32.4 \% \\
\text { (23.0 to } 45.4 \text { ) }\end{array}$ & $\begin{array}{l}2.1 \% \text { more } \\
\text { (7.3 fewer to } 15.1 \text { more) }\end{array}$ & $\begin{array}{l}\oplus \oplus \bigcirc \bigcirc \\
\text { Lowt }\end{array}$ & $\begin{array}{l}\text { Immobilisation in external } \\
\text { rotation does not seem to be } \\
\text { beneficial in prevention of } \\
\text { shoulder redislocations. }\end{array}$ \\
\hline
\end{tabular}


Table 4 Summary of findings for open compared with arthroscopic labrum surgery in treatment of chronic post-traumatic shoulder instability

\begin{tabular}{|c|c|c|c|c|c|c|}
\hline \multicolumn{7}{|c|}{ Open compared with arthroscopic labrum surgery for prevention of recurrent dislocations in treatment of chronic post-traumatic shoulder instability } \\
\hline \multicolumn{7}{|c|}{$\begin{array}{l}\text { Patient or population: chronic post-traumatic shoulder instability in general population } \\
\text { Setting: surgical versus surgical management } \\
\text { Intervention: open labrum surgery } \\
\text { Comparison: arthroscopic labrum surgery }\end{array}$} \\
\hline \multirow{2}{*}{$\begin{array}{l}\text { Outcome } \\
\text { Number of participants } \\
\text { (studies) }\end{array}$} & \multirow{2}{*}{$\begin{array}{l}\text { Relative effect } \\
(95 \% \mathrm{Cl})\end{array}$} & \multicolumn{3}{|c|}{ Anticipated absolute effects $(95 \% \mathrm{Cl})$} & \multirow[b]{2}{*}{ Certainty } & \multirow[b]{2}{*}{ What happens } \\
\hline & & Arthroscopic & Open & Difference & & \\
\hline $\begin{array}{l}\text { Redislocation rate after surgical intervention } \\
\text { in treatment of chronic post-traumatic } \\
\text { shoulder instability } \\
\text { Follow-up: mean } 2 \text { years } \\
\text { Number of participants: } 269 \\
\text { (three RCTs) }\end{array}$ & $\begin{array}{l}\text { RR } 0.43 \\
\text { (0.19 to } 0.97)\end{array}$ & $13.4 \%$ & $\begin{array}{l}5.8 \% \\
(2.6 \text { to } 13.0)\end{array}$ & $\begin{array}{l}7.7 \% \text { fewer } \\
\text { (10.9 fewer to } 0.4 \text { fewer) }\end{array}$ & $\begin{array}{l}\oplus \oplus \bigcirc \bigcirc \\
\text { Low }^{*}\end{array}$ & $\begin{array}{l}\text { Open labrum repair seems to } \\
\text { be beneficial in prevention of } \\
\text { shoulder redislocations. }\end{array}$ \\
\hline
\end{tabular}

*Rated down for serious risk of bias (lack of blinding) and serious imprecision.

$\mathrm{RCT}$, randomised controlled trial; RR, relative risk.

Explanations for the Cochrane Summary of Findings table (http://www.cochranelibrary.com/about/explanations-for-cochrane-summary-of-findings-sof-tables.html)

\section{Restriction band usage}

There was one trial investigating the use of a shoulder motion restriction band after immobilisation in ER (vs immobilisation in ER only). The restriction band had no effect on shoulder redislocation rates. ${ }^{57}$

\section{Chronic post-traumatic shoulder instability}

Open versus arthroscopic surgery

There was a statistically significant difference in favour of open labrum repair for preventing redislocations (three studies, 269 patients) (RR $0.43 ; 95 \%$ CI 0.19 to $0.97 ; \mathrm{p}=0.04 ; \mathrm{I}^{2}=0 \%$; $\mathrm{p}=0.43$ ) and instability (two studies, 223 patients) (RR 0.49; $95 \%$ CI 0.26 to $0.92 ; \mathrm{p}=0.03 ; \mathrm{I}^{2}=0 \% ; \mathrm{p}=0.99$ ) (online supplementary appendix figure $3 \mathrm{a}, \mathrm{b}$; table 4 ). The $\mathrm{NNH}$ was approximately 12 , defined here as a redislocation that would not have occurred if open instead of arthroscopic technique had been used.

\section{Absorbable versus non-absorbable suture anchors, tacks or suture materials}

There were no differences in recurrent instability rates between absorbable and non-absorbable suture anchors (three studies,
232 patients) ${ }^{48-50}$ (RR $0.62 ; 95 \%$ CI 0.21 to $1.86 ; \mathrm{p}=0.40$; $\mathrm{I}^{2}=0 \% ; \mathrm{p}=0.95$ ) (online supplementary appendix figure 4; table 5). The absorbability of tacks or suture material had no effect on recurrent instability rate. ${ }^{48-50} 53-55$

Accelerated versus traditional postsurgical rehabilitation and different surgical procedures

There was one trial investigating each of these topics. Neither the pace of rehabilitation after surgical intervention nor the surgical method had any effect on the redislocations or chronic instability. ${ }^{515256}$

\section{Harms}

Three publications specified and reported harms as a study outcome a priori. ${ }^{5} 6{ }^{44}$ Thirteen publications reported harms occurrence but did not list harms as a prespecified outcome. $^{2-4741-43464749515354}$ Nine publications did not mention harms. ${ }^{15} 164548505255-57$ There were 19 patients (1.5\%) with temporary pain, rigidity or stiffness in the treated shoulder, 17 patients $(1.3 \%)$ with transient nerve injuries, 5 patients $(0.39 \%)$ with superficial wound infections, 3 patients $(0.24 \%)$ with cases

Table 5 Summary of findings for use of absorbable compared with non-absorbable implant materials in labrum surgery in treatment of chronic post-traumatic shoulder instability

\begin{tabular}{|c|c|c|c|c|c|c|}
\hline \multicolumn{7}{|c|}{$\begin{array}{l}\text { Absorbable compared with non-absorbable implant materials in labrum surgery for preve } \\
\text { shoulder instability }\end{array}$} \\
\hline \multicolumn{7}{|c|}{$\begin{array}{l}\text { Patient or population: chronic post-traumatic shoulder instability in general population } \\
\text { Setting: surgical versus surgical management } \\
\text { Intervention: absorbable implant material } \\
\text { Comparison: non-absorbable implant material }\end{array}$} \\
\hline \multirow{2}{*}{$\begin{array}{l}\text { Outcome } \\
\text { Number of participants } \\
\text { (studies) }\end{array}$} & \multicolumn{4}{|c|}{ Anticipated absolute effects $(95 \% \mathrm{Cl})$} & \multirow[b]{2}{*}{ Certainty } & \multirow[b]{2}{*}{ What happens } \\
\hline & $\begin{array}{l}\text { Relative effect } \\
(95 \% \mathrm{Cl})\end{array}$ & $\begin{array}{l}\text { Non- } \\
\text { absorbable }\end{array}$ & Absorbable & Difference & & \\
\hline $\begin{array}{l}\text { Recurrent instability rate after surgical } \\
\text { intervention in treatment of chronic post- } \\
\text { traumatic shoulder instability } \\
\text { Follow-up: mean } 2 \text { years } \\
\text { Number of participants: } 232 \\
\text { (three RCTs) }\end{array}$ & $\begin{array}{l}\text { RR } 0.62 \\
\text { (0.21 to } 1.86 \text { ) }\end{array}$ & $9.6 \%$ & $\begin{array}{l}5.9 \% \\
(2.0 \text { to } 17.8)\end{array}$ & $\begin{array}{l}3.6 \% \text { fewer } \\
\text { (7.6 fewer to } 8.2 \\
\text { more) }\end{array}$ & $\begin{array}{l}\oplus \oplus \oplus \bigcirc \\
\text { Moderate* }^{*}\end{array}$ & $\begin{array}{l}\text { Absorbability of implants } \\
\text { does not seem to affect } \\
\text { the recurrent instability } \\
\text { rate. }\end{array}$ \\
\hline
\end{tabular}


of adhesive capsulitis and 1 patient $(0.08 \%)$ with septic arthritis in the shoulder (online supplementary appendix table 5 ).

\section{DISCUSSION}

Our review aimed to answer two patient-oriented questions relevant to clinical practice: (1) What are the best treatments to reduce the incidence of chronic shoulder instability after a firsttime traumatic shoulder dislocation? and (2) How should the clinician best treat a patient with chronic post-traumatic shoulder instability? Evidence regarding the comparative effectiveness of surgery versus non-surgical treatment is currently available only for a first-time traumatic shoulder dislocation: early surgery led to fewer shoulder redislocations. However, approximately half of the patients primarily treated non-surgically do not experience a shoulder redislocation or develop shoulder instability within 2 years of the primary injury. Immobilisation in ER was not beneficial after a first-time traumatic shoulder dislocation compared with immobilisation in IR.

For the treatment of chronic post-traumatic shoulder instability, we did not identify any RCTs that assessed the effectiveness or efficacy of surgery versus non-surgical measures or sham surgery. Open Bankart repair produced more reliable results than the arthroscopic method for prevention of shoulder redislocations. There were no differences between other technical aspects of surgery.

\section{Treatment after a first-time traumatic shoulder dislocation}

Labrum repair was more effective than non-surgical treatment for first-time dislocation in terms of fewer redislocations. Arthroscopic lavage was no more effective at 2 years compared with non-surgical treatment, making arthroscopic lavage a candidate placebo surgical treatment in this context. Our NMA indicated a beneficial effect of labrum repair on the incidence of shoulder redislocation when compared with non-surgical treatment. The NNT-in this context the number of patients who needed to undergo surgery to prevent one patient having a shoulder redislocation-was 2.5-5.6 based on external data ${ }^{35}$ and 2.0-4.7 based on the data in the included trials. ${ }^{346}$

Regarding disease-specific quality of life, no meaningful meta-analysis was possible. There were some differences between the treatment alternatives in favour of labrum repair (online supplementary appendix table 5). ${ }^{4} 6$ Even though the differences were small, they were consistent with and explained by the reduced risk of shoulder redislocations and chronic instability in the groups treated with labrum repair.

The study populations in the included RCTs consisted mostly of young men and the majority of dislocations were sports related, making the results applicable to the typical patient, but the generalisability to women, non-athletes and older patients is uncertain. Our results are less favourable towards surgery than those presented by previous systematic reviews and meta-analyses. ${ }^{58} 59$ This difference might be attributed to a strict inclusion of RCTs only, the handling of labrum repair, arthroscopic lavage and non-surgical treatment as separate entities and the use of NMA. Overall, since only about half of the patients in the included studies and in a large long-term prospective cohort ${ }^{60}$ suffered shoulder redislocations and the NNTs are relatively high, the prevailing practice ${ }^{17}$ of low-threshold surgery after a first-time shoulder dislocation can be questioned. Previous studies have suggested that delaying surgery and waiting to see whether the patient developed chronic instability after a firsttime traumatic shoulder dislocation did not lead to a less favourable prognosis of instability, quality of life or glenohumeral joint osteoarthritis. ${ }^{61} 62$ Compared with routine surgery after a firsttime traumatic shoulder dislocation, waiting to see if a patient develops chronic instability despite rehabilitation might direct resources more efficiently and may save half of patients from unnecessary surgery.

Arm position after primary traumatic shoulder dislocation (immobilisation in ER vs IR) had no effect on the risk of redislocations or chronic shoulder instability. This finding is consistent with other meta-analyses. ${ }^{63-66}$ The number of unpublished trials investigating immobilisation in ER identified was equal to the number of RCTs included in this review, which raises concerns of publication bias. Immobilisation in an ordinary arm sling for comfort appears to be preferable to the use of an ER brace.

\section{Chronic post-traumatic shoulder instability}

There were no RCTs exploring the effectiveness of surgery versus non-surgical treatment, which is an important evidence gap. Open labrum repair appears to be more effective than arthroscopic labrum repair in successfully stabilising an unstable shoulder, while there was no difference in other outcomes or harms. The point estimate for the RR was 0.43 ; this means that arthroscopic technique was more than twice as likely to fail as open technique, and the $\mathrm{NNH}$ was approximately 12 , defined here as a shoulder redislocation that would not have occurred if open technique had been used instead of arthroscopy. Previous meta-analyses, which have also included non-randomised studies, have suggested no difference between open and arthroscopic techniques ${ }^{67-73}$ or a difference in favour of the open method. ${ }^{74-76}$ Although we have witnessed a rapid increase in the popularity of arthroscopy within the past two decades to the point of it being considered the accepted standard, ${ }^{77-81}$ our findings might justify a tempering of enthusiasm for the arthroscopic method. On the other hand, even though the perceived effectiveness of surgery might be overestimated since the natural course of chronic post-traumatic shoulder instability is largely unknown, the results of surgery for chronic instability are encouraging in terms of the low redislocation rates after surgery in the included trials.

\section{Study bias}

The risk of bias among the included RCTs was relatively low. However, concern for confounding still exists, as there was considerable heterogeneity in the follow-up time and less than half $(41 \%)$ of the studies used any kind of blinding. In most trials, the primary outcome was recurrent dislocation, a relatively 'hard' endpoint. However, in the included RCTs, the definition of redislocation varied from a solely symptoms-based diagnosis to one that was confirmed radiographically or alternatively, an event that required a manual reduction. Analysis on the effect of conflicts of interest or industry sponsorship on trial results was not possible due to heterogeneity.

\section{Limitations}

The use of shoulder instability-validated outcome instruments was rare 6154244454752 and baseline values were seldom reported. ${ }^{454}$ The publications also generally did not meet the assumptions of the power calculations, making a type II error possible. Another limitation is publication bias in the field, as studies with 'negative results' for the intervention are more likely to remain unpublished, ${ }^{82}$ risking overestimation of the true effect in meta-analyses. A methodological limitation is that no sensitivity analyses or meta-regression were performed. Finally, our findings apply only to patients with almost intact 
bony glenoids, as in all but one trial no marked bony defects of the glenoid were present among the patients. ${ }^{52}$ This has direct clinical relevance as shoulder dislocation occasionally causes a sizeable glenoid fracture, or the glenoid bone may erode with numerous redislocations. ${ }^{83}$

A limitation to the clinical relevance of our findings is that the effect of a shoulder redislocation on patient quality of life remains unclear. An occasional shoulder dislocation may result in mild discomfort while the shoulder functions otherwise normally. In contrast, a subjectively unstable shoulder may be associated with constant fear of dislocations, kinesiophobia and poor function even in the absence of redislocations. ${ }^{44}$ If the primary treatment aim was to prevent redislocations only, shoulder arthrodesis would be very effective. However, such a treatment would also likely be associated with poor functional outcomes, and we might reasonably expect this treatment and outcome is not desired by patients. ${ }^{85}$

\section{FUTURE RESEARCH}

The most important evidence gaps regarding clinical practice are the effectiveness of surgery for chronic post-traumatic shoulder instability and what is the ideal physiotherapy regime for non-surgical management after a primary traumatic dislocation or for chronic post-traumatic instability. The impact of surgery to patient quality of life should also be investigated. Future trials should be adequately designed, ${ }^{8687}$ powered and reported, ${ }^{88}$ and use validated measures of disability or function in addition to the shoulder redislocation rate. Due to the placebo effects associated with surgery, further trials should be double blinded and placebo controlled whenever possible. ${ }^{6890}$

\section{CONCLUSIONS}

There was an RR of 0.15 (95\% CI 0.03 to 0.8 ) for a recurrent dislocation after first-time traumatic shoulder dislocation

\section{What is already known on this subject?}

- After a first-time traumatic shoulder dislocation, approximately half of patients developed recurrent shoulder dislocation within 2 years.

- Some studies have shown promising results with surgery after a first-time traumatic shoulder dislocation. Primary surgery has been advocated to prevent chronic shoulder instability.

- Many patients with chronic post-traumatic shoulder instability are offered stabilising surgery. Current practice favours arthroscopic labral repair over open surgery.

\section{What this study adds?}

- There was moderate-quality evidence that patients who had labrum repair surgery after a first-time traumatic shoulder dislocation had lower recurrent dislocation rates than patients treated with physiotherapy. However, surgical treatment has a relatively high number needed to treat (2.55.6) to prevent redislocation.

- In the treatment of chronic post-traumatic shoulder instability, effectiveness of surgery compared with nonsurgical treatment is not known. Low-quality evidence supported open labrum repair over arthroscopic labrum repair. in favour of labrum repair compared with physiotherapy. The certainty in the evidence was moderate. Forty-seven per cent of patients with a first-time traumatic shoulder dislocation treated non-surgically did not experience a redislocation within 2 years.

Acknowledgements We thank Professor Teppo Järvinen for his insightful comments and critical review of the manuscript, information specialist Ritva Miikki for the database searches and Teppo Huttunen and Joni Keto-Tokoi of 4Pharma for assistance with the NMA.

Contributors LK, TL, AM and MP all participated in the conception and design of the study. LK and TL acquired the data. LK performed the analyses and TL, AM and MP participated in interpreting the analyses. TL and LK drafted the manuscript. All authors critically revised and approved the final version of the manuscript.

Funding LK has received a research grant from Finnish Research Foundation for Orthopaedics and Traumatology (FRFOT) and from the Helsinki Academic Medical Centre. TL has received a research grant from the FRFOT.

Disclaimer The funders had no role in any part of the study or in any decision about publication.

Competing interests None declared.

Patient consent Not required.

Provenance and peer review Not commissioned; externally peer reviewed.

Data sharing statement The study protocol is available from the PROSPERO registry, https://www.crd.york.ac.uk/prospero/ with the identifier CRD42015020303. Statistical codes are available for the Netmeta package in program $\mathrm{R}$ (online supplementary appendix 2) and RevMan (online supplementary appendix 3 ). Full data are presented in online supplementary appendix tables $2-7$.

Open access This is an open access article distributed in accordance with the Creative Commons Attribution Non Commercial (CC BY-NC 4.0) license, which permits others to distribute, remix, adapt, build upon this work non-commercially, and license their derivative works on different terms, provided the original work is properly cited and the use is non-commercial. See: http://creativecommons.org/ licenses/by-nc/4.0/

(c) Article author(s) (or their employer(s) unless otherwise stated in the text of the article) 2018. All rights reserved. No commercial use is permitted unless otherwise expressly granted.

\section{REFERENCES}

1 Yang NP, Chen HC, Phan DV, et al. Epidemiological survey of orthopedic joint dislocations based on nationwide insurance data in Taiwan, 2000-2005. BMC Musculoskelet Disord 2011;12:253

2 Fabbriciani C, Milano G, Demontis A, et al. Arthroscopic versus open treatment of Bankart lesion of the shoulder: a prospective randomized study. Arthroscopy 2004;20:456-62.

3 Jakobsen BW, Johannsen HV, Suder P, et al. Primary repair versus conservative treatment of first-time traumatic anterior dislocation of the shoulder: a randomized study with 10-year follow-up. Arthroscopy 2007;23:118-23.

4 Kirkley A, Griffin S, Richards C, et al. Prospective randomized clinical trial comparing the effectiveness of immediate arthroscopic stabilization versus immobilization and rehabilitation in first traumatic anterior dislocations of the shoulder. Arthroscopy: The Journal of Arthroscopic \& Related Surgery 1999;15:507-14.

5 Archetti Netto N, Tamaoki MJ, Lenza M, et al. Treatment of Bankart lesions in traumatic anterior instability of the shoulder: a randomized controlled trial comparing arthroscopy and open techniques. Arthroscopy 2012;28:900-8.

6 Robinson CM, Jenkins PJ, White TO, et al. Primary arthroscopic stabilization for a first-time anterior dislocation of the shoulder. A randomized, double-blind trial. J Bone Joint Surg Am 2008;90:708-21.

7 Wintzell G, Haglund-Akerlind Y, Ekelund A, et al. Arthroscopic lavage reduced the recurrence rate following primary anterior shoulder dislocation. A randomised multicentre study with 1-year follow-up. Knee Surg Sports Traumatol Arthrosc 1999;7:192-6.

8 Bankart ASB. The pathology and treatment of recurrent dislocation of the shoulderjoint. Br J Surg 1938;26:23-9.

9 Bankart AS, Cantab MC. Recurrent or habitual dislocation of the shoulder-joint 1923. Clin Orthop Relat Res 1993:291:3-6.

10 Rowe CR. Prognosis in dislocations of the shoulder. J Bone Joint Surg Am 1956;38A:957-77.

11 Rowe CR, Patel D, Southmayd WW. The Bankart procedure: a long-term end-result study. J Bone Joint Surg Am 1978;60:1-16.

12 Liavaag S, Svenningsen S, Reikerås 0 , et al. The epidemiology of shoulder dislocations in Oslo. Scand J Med Sci Sports 2011;21:e334-e340. 
13 Olds M, Ellis R, Donaldson K, et al. Risk factors which predispose first-time traumatic anterior shoulder dislocations to recurrent instability in adults: a systematic review and meta-analysis. Br J Sports Med 2015;49:913-22.

14 Youm T, Takemoto R, Park BK. Acute management of shoulder dislocations. J Am Acad Orthop Surg 2014;22:761-71

15 Kirkley A, Werstine R, Ratjek A, et al. Prospective randomized clinical trial comparing the effectiveness of immediate arthroscopic stabilization versus immobilization and rehabilitation in first traumatic anterior dislocations of the shoulder: long-term evaluation. Arthroscopy 2005;21:55-63.

16 Wintzell G, Haglund-Akerlind Y, Nowak J, et al. Arthroscopic lavage compared with nonoperative treatment for traumatic primary anterior shoulder dislocation: a 2-year follow-up of a prospective randomized study. J Shoulder Elbow Surg 1999:8:399-402.

17 Balke M, Shafizadeh S, Bouillon B, et al. Management of shoulder instability: the current state of treatment among German orthopaedic surgeons. Arch Orthop Trauma Surg 2016;136:1717-21.

18 Malhotra A, Freudmann MS, Hay SM. Management of traumatic anterior shoulder dislocation in the 17- to 25-year age group: a dramatic evolution of practice. $J$ Shoulder Elbow Surg 2012;21:545-53.

19 Weel H, Tromp W, Krekel PR, et al. International survey and surgeon's preferences in diagnostic work-up towards treatment of anterior shoulder instability. Arch Orthop Trauma Surg 2016;136:741-6.

20 Virk MS, Manzo RL, Cote M, et al. Comparison of time to recurrence of instability after open and arthroscopic bankart repair techniques. Orthop J Sports Med 2016;4:232596711665411.

21 Kachooei AR, Talaei-Khoei M, Faghfouri A, et al. Factors associated with operative treatment of enthesopathy of the extensor carpi radialis brevis origin. J Shoulder Elbow Surg 2016:25:666-70.

22 Liberati A, Altman DG, Tetzlaff J, et al. The PRISMA statement for reporting systematic reviews and meta-analyses of studies that evaluate health care interventions: explanation and elaboration. J Clin Epidemiol 2009;62:e1-e34.

23 Furlan AD, Pennick V, Bombardier C, et al. 2009 updated method guidelines for systematic reviews in the Cochrane Back Review Group. Spine 2009;34:1929-41.

24 Bourgeois FT, Murthy S, Mandl KD. Outcome reporting among drug trials registered in ClinicalTrials.gov. Ann Intern Med 2010;153:158-66.

25 Lexchin J. Sponsorship bias in clinical research. Int J Risk Saf Med 2012;24:233-42.

26 Stelfox HT, Chua G, O'Rourke K, et al. Conflict of interest in the debate over calciumchannel antagonists. N Eng/ J Med Overseas Ed 1998;338:101-6.

27 Mather RC, Orlando LA, Henderson RA, et al. A predictive model of shoulder instability after a first-time anterior shoulder dislocation. J Shoulder Elbow Surg 2011:20:259-66.

28 Rowe CR, Sakellarides HT. Factors related to recurrences of anterior dislocations of the shoulder. Clin Orthop 1961;20:40-8.

29 te Slaa RL, Wijffels MP, Brand R, et al. The prognosis following acute primary glenohumeral dislocation. J Bone Joint Surg Br 2004;86:58-64.

30 te Slaa RL, Brand R, Marti RK. A prospective arthroscopic study of acute first-time anterior shoulder dislocation in the young: a five-year follow-up study. J Shoulder Elbow Surg 2003;12:529-34.

31 Sachs RA, Lin D, Stone ML, et al. Can the need for future surgery for acute traumatic anterior shoulder dislocation be predicted? J Bone Joint Surg Am 2007;89:1665-74

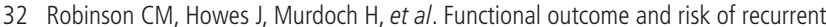
instability after primary traumatic anterior shoulder dislocation in young patients. J Bone Joint Surg Am 2006;88:2326-36.

33 Simonet WT, Cofield RH. Prognosis in anterior shoulder dislocation. Am J Sports Med $1984 ; 12: 19-24$.

34 Andrade C. The numbers needed to treat and harm (NNT, NNH) statistics: what they tell us and what they do not. J Clin Psychiatry 2015;76:e330-e333.

35 Wasserstein DN, Sheth U, Colbenson K, et al. The true recurrence rate and factors predicting recurrent instability after nonsurgical management of traumatic primary anterior shoulder dislocation: a systematic review. Arthroscopy 2016:32:2616-25.

36. Rücker G, Schwarzer G, Krahn U, et al. Network meta-analysis using frequentist methods, 2015.

37 Schünemann HBJ, Guyatt G, Oxman A, GRADE handbook for grading quality of evidence and strength of recommendations: GRADE Working Group, 2013.

38 The Nordic Cochrane Centre, The Cochrane Collaboration. Review Manager (RevMan). Copenhagen, Denmark. 2014.

39 R Core Team. R: a language and environment for statistical computing. 3.2.1. Vienna, Austria: R Foundation for Statistical Computing. 1998.

40 GRADEpro GDT. McMaster University: GRADEpro guideline development tool. 2015.

41 Finestone A, Milgrom C, Radeva-Petrova DR, et al. Bracing in external rotation for traumatic anterior dislocation of the shoulder. J Bone Joint Surg Br 2009;91:918-21.

42 Heidari K, Asadollahi S, Vafaee R, et al. Immobilization in external rotation combined with abduction reduces the risk of recurrence after primary anterior shoulder dislocation. J Shoulder Elbow Surg 2014;23:759-66.

43 Itoi E, Hatakeyama Y, Sato T, et al. Immobilization in external rotation after shoulder dislocation reduces the risk of recurrence. A randomized controlled trial. J Bone Joint Surg Am 2007;89:2124-31.
44 Liavaag S, Brox Jl, Pripp AH, et al. Immobilization in external rotation after primary shoulder dislocation did not reduce the risk of recurrence: a randomized controlled trial. J Bone Joint Surg Am 2011;93:897-904.

45 Whelan DB, Litchfield R, Wambolt E, et al. External rotation immobilization for primary shoulder dislocation: a randomized controlled trial. Clinical Orthopaedics and Related Research $囚 2014: 472: 2380-6$.

46 Sperber A, Hamberg P, Karlsson J, et al. Comparison of an arthroscopic and an open procedure for posttraumatic instability of the shoulder: a prospective, randomized multicenter study. J Shoulder Elbow Surg 2001;10:105-8.

47 Mohtadi NG, Chan DS, Hollinshead RM, et al. A randomized clinical trial comparing open and arthroscopic stabilization for recurrent traumatic anterior shoulder instability: two-year follow-up with disease-specific quality-of-life outcomes. J Bone Joint Surg Am 2014;96:353-60.

48 Milano G, Grasso A, Santagada DA, et al. Comparison between metal and biodegradable suture anchors in the arthroscopic treatment of traumatic anterior shoulder instability: a prospective randomized study. Knee Surg Sports Traumatol Arthrosc 2010;18:1785-91.

49 Tan CK, Guisasola I, Machani B, et al. Arthroscopic stabilization of the shoulder: a prospective randomized study of absorbable versus nonabsorbable suture anchors. Arthroscopy 2006;22:716-20

50 Warme WJ, Arciero RA, Savoie FH, et al. Nonabsorbable versus absorbable suture anchors for open Bankart repair. A prospective, randomized comparison. Am J Sports Med 1999;27:742-6

51 Castagna A, Borroni M, Delle Rose G, et al. Effects of posterior-inferior capsular plications in range of motion in arthroscopic anterior Bankart repair: a prospective randomized clinical study. Knee Surg Sports Traumatol Arthrosc 2009;17:188-94.

52 Salomonsson B, Abbaszadegan $\mathrm{H}$, Revay $\mathrm{S}$, et al. The Bankart repair versus the Putti-Platt procedure: a randomized study with WOSI score at 10-year follow-up in 62 patients. Acta Orthop 2009;80:351-6.

53 Magnusson L, Ejerhed L, Rostgård-Christensen L, et al. A prospective, randomized, clinical and radiographic study after arthroscopic Bankart reconstruction using 2 different types of absorbable tacks. Arthroscopy 2006;22:143-51.

54 Elmlund AO, Kartus J, Rostgård-Christensen L, et al. A 7-year prospective, randomized, clinical, and radiographic study after arthroscopic Bankart reconstruction using 2 different types of absorbable tack. Am J Sports Med 2009;37:1930-7

55 Monteiro GC, Ejnisman B, Andreoli CV, et al. Absorbable versus nonabsorbable sutures for the arthroscopic treatment of anterior shoulder instability in athletes: a prospective randomized study. Arthroscopy 2008;24:697-703.

56 Kim SH, Ha KI, Jung MW, et al. Accelerated rehabilitation after arthroscopic Bankart repair for selected cases: a prospective randomized clinical study. Arthroscopy 2003; 19:722-31.

57 Itoi E, Hatakeyama Y, Itoigawa Y, et al. Is protecting the healing ligament beneficial after immobilization in external rotation for an initial shoulder dislocation? Am J Sports Med 2013;41:1126-32

58. Handoll Helen HG, Al-Maiyah Mohammed A. Surgical versus non-surgical treatment for acute anterior shoulder dislocation. Cochrane Library: John Wiley \& Sons, Ltd, 2004

59 Chahal J, Marks PH, Macdonald PB, et al. Anatomic Bankart repair compared with nonoperative treatment and/or arthroscopic lavage for first-time traumatic shoulder dislocation. Arthroscopy 2012;28:565-75.

60 Hovelius L, Rahme H. Primary anterior dislocation of the shoulder: long-term prognosis at the age of 40 years or younger. Knee Surgery, Sports Traumatology, Arthroscopy 2016:24:330-42.

61 Kavaja L, Pajarinen J, Sinisaari I, et al. Arthrosis of glenohumeral joint after arthroscopic Bankart repair: a long-term follow-up of 13 years. J Shoulder Elbow Surg 2012:21:350-5.

62 Plath JE, Aboalata M, Seppel G, et al. Prevalence of and risk factors for dislocation arthropathy: radiological long-term outcome of arthroscopic bankart repair in 100 shoulders at an average 13-year follow-up. Am J Sports Med 2015;43:1084-90.

63 Hanchard NC, Goodchild LM, Kottam L. Conservative management following closed reduction of traumatic anterior dislocation of the shoulder. Cochrane Database Syst Rev 2014;4:CD004962

64 Whelan DB, Kletke SN, Schemitsch G, et al. Immobilization in external rotation versus internal rotation after primary anterior shoulder dislocation: a meta-analysis of randomized controlled trials. Am J Sports Med 2016:44:521-32.

65 Vavken P, Sadoghi P, Quidde J, et al. Immobilization in internal or external rotation does not change recurrence rates after traumatic anterior shoulder dislocation. J Shoulder Elbow Surg 2014;23:13-19.

66 Liu A, Xue X, Chen Y, et al. The external rotation immobilisation does not reduce recurrence rates or improve quality of life after primary anterior shoulder dislocation: a systematic review and meta-analysis. Injury 2014;45:1842-7.

67 Brophy RH, Marx RG. The treatment of traumatic anterior instability of the shoulder: nonoperative and surgical treatment. Arthroscopy 2009:25:298-304.

68 Hobby J, Griffin D, Dunbar M, et al. Is arthroscopic surgery for stabilisation of chronic shoulder instability as effective as open surgery? A systematic review and metaanalysis of 62 studies including 3044 arthroscopic operations. J Bone Joint Surg Br 2007;89:1188-96. 
69 Godin J, Sekiya JK. Systematic review of arthroscopic versus open repair for recurrent anterior shoulder dislocations. Sports Health 2011;3:396-404.

$70 \mathrm{Ng}$ C, Bialocerkowski A, Hinman R. Effectiveness of arthroscopic versus open surgical stabilisation for the management of traumatic anterior glenohumeral instability. Int J Evid Based Healthc 2007;5:182-207.

71 Petrera M, Patella V, Patella S, et al. A meta-analysis of open versus arthroscopic Bankart repair using suture anchors. Knee Surg Sports Traumatol Arthrosc 2010;18:1742-7.

72 Chen L, Xu Z, Peng J, et al. Effectiveness and safety of arthroscopic versus open Bankart repair for recurrent anterior shoulder dislocation: a meta-analysis of clinical trial data. Arch Orthop Trauma Surg 2015;135:529-38.

73 Pulavarti RS, Symes TH, Rangan A. Surgical interventions for anterior shoulder instability in adults. Cochrane Database Syst Rev 2009;4:CD005077.

74 Mohtadi NG, Bitar IJ, Sasyniuk TM, et al. Arthroscopic versus open repair for traumatic anterior shoulder instability: a meta-analysis. Arthroscopy 2005;21:652-8.

75 Wang L, Liu Y, Su X, et al. A meta-analysis of arthroscopic versus open repair for treatment of bankart lesions in the shoulder. Medical Science Monitor 2015;21:3028-35.

76 Lenters TR, Franta AK, Wolf FM, et al. Arthroscopic compared with open repairs for recurrent anterior shoulder instability. A systematic review and meta-analysis of the literature. J Bone Joint Surg Am 2007;89:244-54.

77 Blomquist J, Solheim E, Liavaag S, et al. Shoulder instability surgery in Norway: the first report from a multicenter register, with 1-year follow-up. Acta Orthop 2012:83:165-70.

78 Owens BD, Harrast JJ, Hurwitz SR, et al. Surgical trends in Bankart repair: an analysis of data from the American Board of Orthopaedic Surgery certification examination. Am J Sports Med 2011;39:1865-9.

79 Wasserstein D, Dwyer T, Veillette C, et al. Predictors of dislocation and revision after shoulder stabilization in Ontario, Canada, from 2003 to 2008. Am J Sports Med 2013:41:2034-40
80 Zhang AL, Montgomery SR, Ngo SS, et al. Arthroscopic versus open shoulder stabilization: current practice patterns in the United States. Arthroscopy 2014;30:436-43.

81 Kavaja L, Malmivara A, Lähdeoja T, et al. Shoulder capsular surgery in Finland between 1999 and 2008: a nationwide register analysis. Scand J Surg 2018;107:172-9.

82 McGauran N, Wieseler B, Kreis J, et al. Reporting bias in medical research - a narrative review. Trials 2010;11:37.

83 Griffith JF, Antonio GE, Yung PS, et al. Prevalence, pattern, and spectrum of glenoid bone loss in anterior shoulder dislocation: CT analysis of 218 patients. AJR Am J Roentgenol 2008;190:1247-54.

84 Tjong VK, Devitt BM, Murnaghan ML, et al. A qualitative investigation of return to sport after arthroscopic bankart repair: beyond stability. Am J Sports Med 2015;43:2005-11.

85 Diaz JA, Cohen SB, Warren RF, et al. Arthrodesis as a salvage procedure for recurrent instability of the shoulder. J Shoulder Elbow Surg 2003;12:237-41.

86 Chan AW, Tetzlaff JM, Altman DG, et al. SPIRIT 2013 Statement: defining standard protocol items for clinical trials. Rev Panam Salud Publica 2015;38:506-14.

87 Chan AW, Tetzlaff JM, Gøtzsche PC, et al. SPIRIT 2013 explanation and elaboration: guidance for protocols of clinical trials. BMJ 2013;346:e7586.

88 Schulz KF, Altman DG, Moher D, et al. CONSORT 2010 statement: updated guidelines for reporting parallel group randomised trials. BMJ 2010;340:c332.

89 Moseley JB, O'Malley K, Petersen NJ, et al. A Controlled trial of arthroscopic surgery for osteoarthritis of the knee. N Engl J Med Overseas Ed 2002;347:81-8.

90 Sihvonen R, Paavola M, Malmivaara A, et al. Arthroscopic partial meniscectomy versus sham surgery for a degenerative meniscal tear. $N$ Engl J Med 2013;369:2515-24.

91 Franchignoni F, Vercelli S, Giordano A, et al. Minimal clinically important difference of the disabilities of the arm, shoulder and hand outcome measure (DASH) and its shortened version (QuickDASH). J Orthop Sports Phys Ther 2014;44:30-9. 